\title{
Soft Control Systems on Lie Groups
}

\section{Akyar B and Kara Hansen A*}

Department of Mathematics, Aalborg University, Denmark

\begin{abstract}
In this work, we apply soft theory to control theory. With the inspiration of soft theory we define soft Lie groups and construct soft control systems on Lie groups with observation. For the decision argument, we use some observability characterization and give an example.
\end{abstract}

Keywords: Soft sets; Soft Lie groups; Soft control systems; Observability

\section{Introduction}

The aim of this paper is to study soft theory from the mathematical control theory point of view. For this purpose, we introduce soft Lie group concept and other related topics from Lie theories which are useful to construct soft control systems. We would like to construct soft control systems in order to use observability property which is one of the fundamental classical problem in control theory that helps to turn uncertainity into certainity by the given characterizations in ref. [1,2].

Soft theory begins with the soft sets in its history and soft sets were originally introduced by Pawlak in 1993 which is motivated by Molodtsov in 1999 who presented the fundamentals of the soft theory with some of its applications as a new parametrized mathematical tool in order to deal with uncertainity avoiding some difficulties of the inadequacy of the parametrization tool of the theory. So, one can approach many problems in engineering, economics, medicine and sociology applying the soft theory into several directions [3].

Soft set is an instrument for dealing with uncertainity problems. Its efficiency in dealing with uncertainity problems is as a result of its parametrized concept.

Soft theory has been studied increasingly by many various mathematicians, especially the algebraic structure of soft set theory and the foundation of the soft topology with the related properties have been studied in more details by Acar-Koyuncu-Tanay and Cagman et al. $[4,5]$.

The importance of soft theory can be seen in some applications containing uncertainties, especially in decision making problems. In order to handle such uncertainties, we would like to introduce soft control systems on Lie groups and carry this problem to the control systems with the aid of soft theory.

There is an advantage of working with soft theory, since the original description of its objects has an approximate nature and there is no need to introduce the notion of exact solution. Avoiding any restriction on the approximate description in soft set theory makes this theory very convenient and easily applicable in practice. The uncertainties appearing in classical methods may be of various types.

We aim to apply soft theory into control theory and see how a soft set appears in control theory in the view of Lie groups. Since soft theory has wide potential for practical applications in various areas. We follow the works of Molodtsov and Maji et al. [6-8], for some basic definitions and related constructions with appropriate notations on soft theory in the first section.

Recently, Shabir and Naz in ref. [9] introduced the notion of soft topological spaces defined over an initial universe with a fixed set of parameters.

They also studied some of basic concepts of soft topological spaces.

This work has two main parts. First part is to introduce soft Lie groups and the second part is for soft control systems.

Let us take $\mathcal{U}$ be an initial universe set and $E$ a set of parameters, where each parameter may be a word or a sentence. Let $\mathcal{P}(\mathcal{U})$ denote the power set of $\mathcal{U}$ and $A$ a subset of $E$.

\section{Definition (Soft set)}

A soft set $(F, A)$ over $\mathcal{U}$ is a parametrized family of subsets of $\mathcal{U}$, where $F: A \rightarrow \mathcal{P}(\mathcal{U})$ is a set-valued mapping called the approximate function of $(F, A)$.

The set of all soft sets over $\mathcal{U}$ is denoted by $S(\mathcal{U})_{A}$. Soft sets can be regarded as neighborhood systems.

\section{Definition (Null-soft set)}

A soft set $(F, A)$ over $\mathcal{U}$ is said to be a null soft set denoted by $\tilde{\emptyset}_{A}$ if $\forall x \in A, F(x)=\varnothing$; (null-set).

Example 1: Let $(X ;)$ be a topological space. The family of all open neighborhoods $T(x)=\{V \in \tau \mid x \in V\} \mathrm{g}$ of a point $x$, is the soft set $(T, X)$ over $X$.

Example 2: Let $\mathcal{U}$ be the set of houses under consideration, and let $W$ be the set of decision parameters. We can $W$ as $W=\left\{w_{1}=\right.$ expensive; $w_{2}=$ beautiful; $w_{3}=$ cheap; $w_{4}=$ big; $w_{5}=$ in good repair; $w_{6}=$ in good view; $w_{7}=$ with big garden $\}$

Let the soft set $(F, A)$ describe the attractiveness and the cost of the houses. Suppose that $\mathcal{U}=\left\{h_{1}, h_{2}, h_{3}, h_{4}, h_{5}, h_{6}\right\}$ and $A=\left\{w_{1}, w_{2}, w_{3}, w_{4}, w_{5}\right\}$.

We can consider the mapping $F$ by "houses (.)", where (.) is to be filled in by one of the parameters $w_{i} \in W$. For instance, $F\left(w_{1}\right)$ means "houses (expensive)", and its functional value is the set $\{e \in \mathcal{U} \mid e$ is an expensive house\}.

*Corresponding author: Kara Hansen A, Department of Mathematics, Aarhus University, Denmark, Tel: (+45)60417231; E-mail: aysekara66@gmail.com; akhansen@ase.au.dk

Received July 19, 2017; Accepted July 27, 2017; Published July 30, 2017

Citation: Akyar B, Kara Hansen A (2017) Soft Control Systems on Lie Groups. J Generalized Lie Theory Appl 11: 273. doi: 10.4172/1736-4337.1000273

Copyright: () 2017 Akyar B, et al. This is an open-access article distributed under the terms of the Creative Commons Attribution License, which permits unrestricted use, distribution, and reproduction in any medium, provided the original author and source are credited. 
Thus we can view $(F, A)$ as a collection of approximate description of an object and the soft set $(F, A)=\left\{\left(w_{i} f_{A}\left(w_{i}\right)\right) \mid w_{i} \in A ; f A(w i) \in P(\mathcal{U}) ; A \subset W\right\}$ describes the attractiveness of houses. Suppose that $F\left(w_{1}\right)=\left\{e_{2}, e_{4}\right\}$, $F\left(w_{2}\right)=\left\{e_{1}, e_{3}\right\}, F\left(w_{3}\right)=\left\{e_{6}\right\}, F\left(w_{4}\right)=\left\{e_{1}, e_{3}, e_{5}\right\}, F\left(w_{5}\right)=\left\{e_{1}\right\}$. We have

$(F, A)=\left\{\left(\right.\right.$ expensive houses, $\left.\left\{e_{2}, e_{4}\right\}\right)$, (beautiful houses, $\left.\left\{\mathrm{e}_{1}, \mathrm{e}_{3}\right\}\right)$, (cheap houses, $\left.\left\{e_{6}\right\}\right)$, (big houses, $\left\{e_{1}, e_{3}, e_{5}\right.$ ), (in good repair houses; $\left.\left.\left\{e_{1}\right\}\right)\right\}$.

Example 3: Let $\mathcal{U}$ be the set of some parts of our body under consideration. Let $W$ be the set of parameters (types of sport). Suppose that

$\mathcal{U}=\left\{b_{1}=\right.$ legs, $b_{2}=$ chest,$b_{3}=$ shoulders $b_{4}=$ back, $b_{5}=$ stomach, $b_{6}=$ hips, $b_{7}=$ bottom, $b_{8}=$ waist $\}$

$W=\left\{w_{1}=\right.$ body fit, $w_{2}=$ running, $w_{3}=$ yoga,$w_{4}=$ swimming, $w_{5}=$ step, $w_{6}=$ zumba, $w_{7}=$ stretching $\}$

Suppose that $F\left(w_{1}\right)=\left\{b_{1}, b_{2}, b_{3}, b_{4}, b_{5}, b_{6}\right\}, F\left(w_{2}\right)=\left\{b_{1}\right\}, F\left(w_{3}\right)=\emptyset$, $F\left(w_{4}\right)=\left\{b_{1}, b_{3}, b_{4}, b_{7}\right\}, F\left(w_{5}\right)=\left\{b_{1}\right\}, F\left(w_{6}\right)=\left\{b_{1}, b_{6}, b_{7}, b_{8}\right\}, F(w 7)=\left\{b_{4}, b_{5}, b_{6}\right.$, $\left.b_{7}, b_{8}\right\}$. We have

$(F, W)=\left\{\left(\right.\right.$ body fit, $\left.\left\{b_{1}, b_{2}, b_{3}, b_{4}, b_{5}, b_{6}\right\}\right)$, (running, $\left.\left\{b_{1}\right\}\right)$, (yoga, $\left.\left\{b_{4}, b_{5}\right\}\right)$,

(swimming, $\left\{b_{1}, b_{3}, b_{4}, b_{7}\right\}$ ), (step, $\left\{b_{1}\right\}$ ), (zumba, $\left\{b_{1}, b_{6}, b_{7}, b_{8}\right\}$ ),

(stretching, $\left.\left.\left\{b_{4}, b_{5}, b_{6}, b_{7}, b_{8}\right\}\right)\right\}$.

Here $(F, W)$ describes the health benefits of sport and physical activity.

\section{Definition (Absolute soft set)}

A soft set $(F, A)$ over $\mathcal{U}$ is said to be an absolute set, denoted by $\mathcal{U}_{A}$, if $\forall x \in A, F(x)=\mathcal{U}$. Clearly,

$$
\mathcal{U}_{A}^{c}=\tilde{\emptyset}_{A} \text { and } \tilde{\emptyset}_{A}^{c}=\mathcal{U}_{A}
$$

\section{Definition (Soft subset)}

Let $\left(F_{1}, A\right)$ and $\left(F_{2}, A\right)$ be two soft sets over $\mathcal{U}$, then $\left(F_{1}, A\right)$ is said to be a soft subset of $\left(F_{2}, A\right)$ if $F_{1}(x) \subseteq F_{2}(x)$, for all $x \in A$ which is denoted by $F_{1} \tilde{\subseteq} F_{2}$.

$\left(F_{1}, A\right)$ is said to be soft equal to $\left(F_{2}, A\right)$ if $F_{1}(x)=F_{2}(x)$, for all $x \in A$. It is denoted by $\left(F_{1}, A\right) \cong\left(F_{2}, A\right)$.

The complement of a soft set $(F, A)$ is defined as $(F, A)^{c}=\left(F^{c}, A\right)$, where $F^{c}(x)=(F(x))^{c}=\mathcal{U}-F(x)$, for all $x \in A$.

Definition: The intersection of a nonempty family of soft sets $\left\{\left(F_{i}, A\right) \mid i \in I\right\}$ over $U$ is defined by

$$
\tilde{\cap}_{i \in I}\left(F_{i}, A\right)=\left(\tilde{\cap}_{i \in I} F_{i}, A\right)
$$

where $\left(\tilde{\cap}_{i \in I} F_{i}\right)(x)=\cap_{i \in I}(F(x))$, for all $x \in A$.

Definition: The union of a nonempty family of soft sets $\left\{\left(F_{i}, A\right) \mid i \in I\right\}$ over $\mathrm{U}$ is defined by

$$
\tilde{\cup}_{i \in I}\left(F_{i}, A\right)=\left(\tilde{\cup}_{i \in I} F_{i}, A\right) \text {. }
$$

Where $\left(\tilde{\cup}_{i \in I} F_{i}\right)(x)=\cup_{i \in I}(F(x))$, for all $x \in A$.

Zorlutuna et al., and Shabir-Naz [9,10], studied on soft topological spaces and introduced the concepts of interior, soft interior, soft neighborhoods, soft continuity and soft compactness.

Definition: Let $\tau$ be a collection of soft sets over a universe $\mathcal{U}$ with a fixed set A of parameters, then $\tau \subseteq S(\mathcal{U})_{A}$ is called a soft topology on $\mathcal{U}$ with a fixed set $A$ if,
$T_{1}: \emptyset_{A}, \mathcal{U}_{A}$ belong to $\tau$.

$T_{2}$ : the union of any number of soft sets in $\tau$ belongs to $\tau$.

$T_{3}$ : the intersection of any two soft sets in $\tau$ belongs to $\tau$.

The triplet $(\mathcal{U}, \tau, A)$ is called soft topological space over $\mathcal{U}$. The members of $\tau$ are called soft open sets in $U$ and their complements are called soft closed sets in $\mathcal{U}$.

Example 4: Consider $\mathcal{U}$ as the set of some parts of our body under consideration and $W$ as the set of parameters as in example 3 and write a soft topology on $\mathcal{U}$.

Let $A=\left\{w_{1}, w_{3}\right\}$ and $F\left(w_{1}\right)=\left\{b_{2}, b_{3}, b_{4}, b_{7}\right\}, F\left(w_{3}\right)=\left\{b_{1}, b_{5}\right\}$ then $(F, A)=\left\{\left(w_{1}, F\left(w_{1}\right)\right),\left(w_{3}, F\left(w_{3}\right)\right)\right\}$.

Let $B=\left\{w_{2}, w_{3}, w_{5}, w_{6}\right\}$ and $G\left(w_{2}\right)=\left\{b_{5}, b_{7}, b_{8}\right\}, G\left(w_{3}\right)=\left\{b_{1}, b_{5}, b_{8}\right\}$, $G\left(w_{5}\right)=\left\{b_{1}, b_{8}\right\}, G\left(w_{6}\right)=\left\{b_{1}, b_{7}\right\}$ then $(G, B)=\left\{\left(w_{2}, G\left(w_{2}\right)\right), \quad\left(w_{3}, G\left(w_{3}\right)\right)\right.$, $\left.\left(w_{5}, G\left(w_{5}\right)\right),\left(w_{6}, G\left(w_{6}\right)\right)\right\}$.

Let $C=\left\{w_{3}\right\}$ and $H\left(w_{3}\right)=\left\{b_{1}, b_{5}\right\}$ then $(C, H)=\left\{\left(w_{3}, H\left(w_{3}\right)\right)\right\}$ and let $D=\left\{w_{1}, w_{2}, w_{3}, w_{5}, w_{6}\right\}$ and $K\left(w_{1}\right)=\left\{b_{2}, b_{3}, b_{4}, b_{7}\right\}, K\left(w_{2}\right)=\left\{b_{5}, b_{7}, b_{8}\right\}$, $K\left(w_{3}\right)=\left\{b_{1}, b_{5}, b_{8}\right\}, K\left(w_{5}\right)=\left\{b_{1}, b_{8}\right\}, K\left(w_{6}\right)=\left\{b_{1}, b_{7}\right\}$ then $(K, D)=\left\{\left(w_{1}, K\left(w_{1}\right)\right)\right.$, $\left.\left(w_{2}, K\left(w_{2}\right)\right),\left(w_{3}, K\left(w_{3}\right)\right),\left(w_{5}, K\left(w_{5}\right)\right),\left(w_{6}, K\left(w_{6}\right)\right)\right\}$.

The family

$\tau=\left\{\varnothing_{W}, \mathcal{U}_{W},(F, A),(G, B),(H, C),(K, D)\right\}$,

is a soft topology, because

$(F, A) \tilde{\cap}(G, B)=(H, C)$

$(F, A) \tilde{\cup}(G, B)=(H, D)$

Aktas-Cagman [11,12] have introduced a soft group as a parametrized family of subgroups and a basic notion of soft group theory and moreover operations on soft groups including intersection, union, Cartesian product, soft subgroup etc. Nazmul-Samanta [13] were motivated in ref. [9] and introduced a notion of soft topological soft groups.

Let $G$ be a group, $A$ any nonempty set and $R$ an arbitrary binary relation between an element of $A$ and an element of $G$.

Let us explain what we mean by correspondence and $F(x)$ is a set valued function $F: A \rightarrow P(G)$ which can be defined as

$F(x)=\{y \in G \mid(x, y) \in R, x \in A, y \in G\}$.

The par $(F, A)$ is a soft set over $G$. Defining a set-valued function from $A$ to $G$ defines a binary relation $R$ on $A \times G$, given by $R=\{(x, y) 2$ $A \times G \mid y \in F(x)\}$.

The triplet $(A, G, R)$ is an approximation set.

Definition: (Soft Group) $A$ soft set $(F, A)$ over a group $G$ is said to be a soft group over $G$ if and only if $F(x)<G$ for all $x \in A$.

Example 1: Suppose that $G=A=S 3=\{e,(12),(13),(23),(123),(132)\}$ and define $F(x)=\left\{y \in G \mid x R y \Leftrightarrow y=x^{n}, n \in \mathbb{N}\right\}$.

The soft group $(F, A)$ is a parametrized family $\{F(x) \mid x \in A\}$ of subsets, which gives us a collection of subgroups of $G$.

$$
\begin{aligned}
& F(e)=\{e\} \\
& F(12)=\{e ;(12)\} \\
& F(13)=\{e,(13)\} \\
& F(23)=\{e,(23)\} \\
& F(123)=\{e,(123),(132)\}=F(132)
\end{aligned}
$$


Note: One cannot produce a soft group for every soft set. Consider $G=S_{3}$ and $H(x)=\{y \in G \mid x R y \Leftrightarrow y \circ(x)=\circ(y)\}$, where $\circ(x)$ is order of $x$ in $G$, then $(H, G)$ is not a soft group over $G$.

Theorem: Let $\left\{\left(F_{i}, A\right) \mid i \in I\right\}$ be a nonempty family of soft groups of $G$ then $\tilde{\cap}_{i \in I}\left(F_{i}, A\right)$ is a soft group over $G$.

Theorem: Let $\left(F_{i}, A\right)$ and $(H, B)$ be two soft groups over $G$. If $A \cap B=\varnothing$, then $\left(F_{i}, A\right) \tilde{\cup}(H, B)$ is a soft group over $G$.

Definition: Let $\left(F_{i}, A\right)$ be a soft group over $G$ and then $\left(F_{i}, A\right)$ is said to be an identity soft group over $G$ if $F(x)=\{\mathrm{e}\}$ for all $x \in A$, where $e$ is the identity element of $G$.

Definition: Let $\left(F_{i}, A\right)$ and $(H, B)$ be two soft groups over $G$. Then $(H, B)$ is a soft subgroup of $(F, A),(H, B) \tilde{<}(F, A)$, if

1) $B \subset A$

2) $H(x)<F(x)$ for all $x \in B$.

Definition: Let $(F, A)$ and $(H, B)$ be two soft groups over $G$ and $K$, respectively. The product of soft groups $(F, A)$ and $(H, B)$ is defined as $(F, A)(H, B)=(U, A \times B)$, where $U(x, y)=F(x) \times H(y)$ for all $(x, y) \in A \times B$.

Theorem: Let $(F, A)$ and $H, B$ be two soft groups over $G$ and $K$, respectively. Then the product $(F, A) \times(H, B)$ is a soft group over $G \times K$.

\section{Soft Lie Group}

Though soft groups and soft topological spaces have been studied, soft Lie groups have not been introduced. Akram and Feng [14] have introduced soft Lie subalgebras.

We aim to see the role of soft theory in Lie theory.

The purpose of this section is to introduce soft Lie groups and related topics in order to study observability decision through soft control systems which is our interest to introduce in this work and it will appear in the next section. For this aim, first we define soft Lie groups related to their soft Lie algebras.

Through this paper we consider connected Lie groups in order to construct soft Lie groups for the nature of the control systems. In order to have observability property, it is essential to assume connectedness.

Let us take a connected Lie group $G$ and its Lie algebra $L(G)$. A soft Lie subalgebra or simply soft Lie algebra is defined by a couple $(\mathrm{A}, \mathrm{f})$, where $A$ is a subset of IR that is the set of parameters and $\mathrm{f}$ is a correspondence between $A$ and the power set $P(L(G))$ such that for $x \in A, f(x) \in P(L(G))$ is a Lie subalgebra of $L(G)$.

Definition: A soft Lie group $(A, G, F)$ is defined as a corresponding soft Lie group of the soft Lie algebra $(A, L(G), f)$ satisfying $\mathrm{F}$ is a correspondence between $A$ and $P(G)$ such that for $x \in A, F(x) \in P(G)$ is a Lie subgroup of $G$.

One can define a special soft Lie group by sending parameters to the simply connected Lie subgroups which are the unique for their Lie algebras.

Definition: A soft derivation algebra is a triplet $\left(A, \operatorname{Der}(L(G)), F_{\mathrm{c}}\right)$, where $F_{\partial}$ is a correspondence between $A \subset \mathrm{IR}$ and $P(\operatorname{Der}(L(G)))$ the power set of $\operatorname{Der}(L(G))$ such that for $x \in A, F_{\partial}(x) \in P(\operatorname{Der}(L(G)))$ is a subderivation and in this case it means a subset of $\operatorname{Der}(L(G))$. Here,

$\operatorname{Der}(L(G))=\{D \in \operatorname{End}(L(G)) \mid D[X, Y]=[D(X), Y]+[X, D(Y)], \forall X$, $Y L(G)\}$.
There is a direct connection between a soft Lie algebra and its soft Lie group by soft exponential correspondence and soft derivative. Soft exponential correspondence sends each Lie subalgebra to its Lie groups which is a Lie subgroup of G. Soft derivation connects each Lie subgroup to its Lie algebra which is a Lie subalgebra of $L(G)$.

Example 2: Consider the Heisenberg Lie group of dimension 3

$$
H=\left\{\left(\begin{array}{ccc}
1 & a & c \\
0 & 1 & b \\
0 & 0 & 1
\end{array}\right) \mid a, b, c \in \mathbb{I R}\right\}
$$

and its Lie algebra is

$$
L(H)=\left\{\left(\begin{array}{ccc}
0 & x & z \\
0 & 0 & y \\
0 & 0 & 0
\end{array}\right) \mid x, y, z \in \mathbb{R}\right\}
$$

Where

$$
\operatorname{span}=\left\{X=\left(\begin{array}{lll}
0 & 1 & 0 \\
0 & 0 & 0 \\
0 & 0 & 0
\end{array}\right), Y=\left(\begin{array}{lll}
0 & 0 & 0 \\
0 & 0 & 1 \\
0 & 0 & 0
\end{array}\right), Z=\left(\begin{array}{lll}
0 & 0 & 1 \\
0 & 0 & 0 \\
0 & 0 & 0
\end{array}\right)\right\}
$$

$[X, Y]=Z$

Here,

$[X, Y]=X Y-Y X$.

The soft Heisenberg Lie algebra (IR, $\left.L(H), f_{L(H)}\right)$ is defined by the following construction:

$f_{L(H)}: \mathrm{IR}^{3} \rightarrow P(L(H))$

such that

$$
\begin{aligned}
& f_{L(H)}(0,0,0)=\left\{\left(\begin{array}{lll}
0 & 0 & 0 \\
0 & 0 & 0 \\
0 & 0 & 0
\end{array}\right)\right\} \\
& f_{L(H)}(0, y, 0)=\left\{\left(\begin{array}{lll}
0 & 0 & 0 \\
0 & 0 & 0 \\
0 & 0 & 0
\end{array}\right) \mid y \in \mathbb{I R}\right\}
\end{aligned}
$$

and

$$
f_{L(H)}(x, y, z)=\left\{\left(\begin{array}{ccc}
0 & x & z \\
0 & 0 & y \\
0 & 0 & 0
\end{array}\right) \mid x, y, z \in \mathbb{R}\right\}
$$

Corresponding soft Heisenberg group (IR, $\left.H, F_{H}\right)$ is

$F_{H}: \mathrm{IR}_{3} \rightarrow P(H)$

such that

$$
\begin{aligned}
& F_{H}(0,0,0)=\left\{\left(\begin{array}{lll}
1 & 0 & 0 \\
0 & 1 & 0 \\
0 & 0 & 1
\end{array}\right)\right\}, \\
& f_{L(H)}(0, y, 0)=\left\{\left(\begin{array}{lll}
1 & 0 & 0 \\
0 & 1 & y \\
0 & 0 & 1
\end{array}\right) \mid y \in \operatorname{IR}\right\}
\end{aligned}
$$

and 


$$
f_{L(H)}(x, y, z)=\left\{\left(\begin{array}{lll}
0 & x & z \\
0 & 1 & y \\
0 & 0 & 1
\end{array}\right) \mid x, y, z \in \mathbb{I R}\right\} .
$$

And, the derivation algebra has the following form:

$$
\operatorname{Der}(L(H))=\operatorname{span}\left\{\begin{array}{l}
D_{1}=\left(\begin{array}{lll}
0 & 0 & 0 \\
0 & 0 & 0 \\
0 & 1 & 0
\end{array}\right), D_{2}=\left(\begin{array}{ccc}
0 & 0 & 0 \\
0 & 0 & 0 \\
-1 & 0 & 0
\end{array}\right), D_{3}=\left(\begin{array}{lll}
1 & 0 & 0 \\
0 & 0 & 0 \\
0 & 0 & 1
\end{array}\right), \\
D_{4}=\left(\begin{array}{lll}
0 & 1 & 0 \\
0 & 0 & 0 \\
0 & 0 & 0
\end{array}\right), D_{5}=\left(\begin{array}{lll}
0 & 0 & 0 \\
1 & 0 & 0 \\
0 & 0 & 0
\end{array}\right), D_{6}=\left(\begin{array}{lll}
0 & 0 & 0 \\
0 & 1 & 0 \\
0 & 0 & 1
\end{array}\right)
\end{array}\right\} .
$$

By the power set of $\operatorname{Der}(L(G))$, soft derivation algebra can be constructed.

Example 3: Consider the following solvable Lie group of dimension 2:

$$
G=\left\{\left(\begin{array}{cc}
x_{1} & x_{2} \\
0 & 1
\end{array}\right) \mid x_{1}, x_{2} \in \mathrm{IR}\right\}
$$

with group operation

$(s, t)\left(x_{1}, x_{2}\right)=\left(s x_{1}, t+s x_{2}\right)$

and its Lie algebra $L(G)$ is given by the following vector fields:

$$
X=\frac{\partial}{\partial x_{1}} \text { and } \mathrm{Y}=e^{\mathrm{x}_{1}} \frac{\partial}{\partial x_{2}}
$$

where $[X, Y]=Y$.

Soft Lie algebra

$f: \mathrm{IR}^{2} \rightarrow P(L(G))$

is defined by

$$
\begin{aligned}
& f(0,0)=\left\{\left(\begin{array}{ll}
0 & 0 \\
0 & 0
\end{array}\right)\right\} \\
& f\left(0, d_{2}\right)=\left\{\left(\begin{array}{ll}
0 & 0 \\
0 & d_{2}
\end{array}\right) \mid d_{2} \in \mathrm{IR}\right\}
\end{aligned}
$$

and

$$
f\left(d_{1}, d_{2}\right)=\left\{\left(\begin{array}{cc}
0 & 0 \\
d_{1} & d_{2}
\end{array}\right) \mid d_{1}, d_{2} \in \mathrm{IR}\right\} .
$$

Its corresponding soft Lie group is

$$
F: \mathrm{IR}^{2} \rightarrow P(G)
$$

is defined by

$$
\begin{aligned}
& F(0,0)=\left\{\left(\begin{array}{ll}
1 & 0 \\
0 & 1
\end{array}\right)\right\}, \\
& F\left(0, x_{2}\right)=\left\{\left(\begin{array}{cc}
1 & x_{2} \\
0 & 1
\end{array}\right) \mid x_{2} \in \mathbb{I R}\right\}
\end{aligned}
$$

and

$$
F\left(x_{1}, x_{2}\right)=\left\{\left(\begin{array}{cc}
x_{1} & x_{2} \\
0 & 1
\end{array}\right) \mid x_{1}, x_{2} \in \mathrm{IR}\right\}
$$

And, the derivation algebra is

$$
\operatorname{Der}(L(G))=\left\{\left(\begin{array}{cc}
0 & 0 \\
d_{1} & d_{2}
\end{array}\right) \mid d_{1}, d_{2} \in \mathrm{IR}\right\} .
$$

By the power set of $\operatorname{Der}(L(G))$, soft derivation algebra can be constructed.
In ref. [14] Akram and Feng introduce the concept of soft intersection Lie subalgebras. In the following, we introduce the soft intersection Lie groups in the same way.

Definition: Let $G$ be a Lie group, $A$ be a subset of $G$ and $(A, \mathcal{U}$, $\left.F_{A}\right)$ be a soft set over a universal set $\mathcal{U}$. Then, $(A, G, F)$ is called a soft intersection Lie group over $\mathcal{U}$ if it satisfies:

(1) $F\left(g_{1} \circ g_{2}\right) F\left(g_{1}\right) \cap F\left(g_{2}\right), \forall g_{1}, g_{2} \in A$,

(2) $F(r g) F(g), \forall g \in G$ and $\forall r \in I R$.

Example: Assume that $\mathcal{U}=\mathrm{IR}_{3}$ is the universal set and $E=H$ is the Heisenberg group of dimension 3. Consider

$$
A=\left\{\left(\begin{array}{lll}
1 & x & 0 \\
0 & 1 & 0 \\
0 & 0 & 1
\end{array}\right) \mid x \in \mathrm{IR}\right\} \subset H
$$

with the soft set $\left(A, \mathcal{U}, F_{A}\right)$, where

$$
F_{A}\left(\begin{array}{lll}
1 & 0 & 0 \\
0 & 1 & 0 \\
0 & 0 & 1
\end{array}\right)=\mathrm{IR}^{3}
$$

and

$$
F_{A}\left(\begin{array}{lll}
1 & x & 0 \\
0 & 1 & 0 \\
0 & 0 & 1
\end{array}\right)=(0, x, 0) \text {. }
$$

We can say that intersection and union of the soft intersection Lie groups are still soft intersection Lie groups.

\section{Soft Control Systems on Lie Groups}

A control system on a Lie group $G$ with observation is a four tuple $\sum=(G, \mathcal{D}, h, S)$, where $\mathrm{G}$ and $\mathrm{S}$ are Lie groups, $\mathrm{h}$ is a smooth function between $\mathrm{G}$ and $\mathrm{S}$, and $\mathcal{D}$ is the dynamic of the system which consists of smooth vector fields parametrized by the controls. Here, $\mathrm{G}$ is called state space, $\mathrm{S}$ is called observation space and $\mathrm{h}$ is called observation function of the system. Depending on the nature of the elements of the dynamic $\mathcal{D}$, control system $\sum$ becomes invariant, bilinear, linear or affine.

Definition: Let $A \subset I R$ be the set of parameters and denote by $P(\Sigma)$ the power set of $\sum$. A soft control system on a Lie group $\mathrm{G}$ with observation is a couple $\left(A, f_{\Sigma}\right)$, where $f_{\Sigma}$ is a correspondence between $A$ and $P\left(\sum\right)$ such that for $x \in \mathrm{A}, f_{\Sigma}(x) \in P\left(\sum\right)$ is a sub control system of $\sum$. Here, we mean all elements of the power set $P(\Sigma)$ are sub control systems.

Remark: If we consider $\sum$ on a soft Lie group, then we have a soft control system which is weaker than the usual soft control system on a Lie group. Here, weakness is the number of choices for the correspondence.

Example: Let us consider a soft control system on $G L(n, I R)$ with observation. For $G L(n, I R)$, we have a number of Lie subgroups such as $S L(n, \mathrm{IR})$, Heisenberg Lie group, diagonal groups, etc. A control system on soft $G L(n, \mathrm{IR})$ Lie group would has less differential equations then the usual soft control system on GL(n, IR). Because, the usual one has all subsystems and the soft control system on soft $G L(n$, IR) Lie group has subsystems with the state spaces which are only Lie subgroups of $G L(n, \mathrm{IR})$. 
Definition: $\left(A, f_{\Sigma}\right)$ is called soft linear control system on a Lie group $\mathrm{G}$ with observation, if the dynamic $\mathcal{D}$ is given by the following family of differential algebra:

$$
\dot{x}(t)=X(x)+\sum_{j=1}^{d} u_{j}(t) \mid Y^{j}(x)
$$

where $x \in G, Y^{1}, \ldots, Y^{d} \in L(G)$ and $X \in I a(G) \subset \operatorname{Der}(L(G))$, i.e.; $X$ is a linear vector field (an element of infinitesimal automorphisms defined on $G$ ) which means that $\mathrm{X}$ induces a 1-parameter of automorphism $X_{t}$ on $G$.

There is an observability characterization for linear control systems on Lie groups given by Ayala-Hacibekiroglu, [1]. In this work, we would like to use this characterization in order to construct observable soft linear control systems. Observability property gives us to distinguish the elements of the state space G just by looking the images of the solutions on $\mathrm{G}$ under observation function $\mathrm{h}$.

Main characterization is in the following.

Theorem [1]: Let $\sum=(G, \mathcal{D}, h, S)$ be a linear control system on a connected Lie group $\mathrm{G}$ with a Lie group homomorphism $\mathrm{h}$ as an observation function. Then, the system $\sum$ is observable if it is locally observable and the intersection between the fixed points set and the kernel of the observation function just consists of the neutral element $e$. Here, the fixed points set is

$$
\operatorname{Fix}(T)=\left\{g \in G \mid X_{t}(g)=g, \forall t \in \mathrm{IR}\right\} .
$$

And, local observability is satisfied by setting null the Lie algebra of the indistinguishable points from the neutral element $e$.

With this argument we have the second information given in ref. [2] by Juoan which tells us when we can have observability in such a system. We use all this information in the following example as a decision process.

Example: Let us consider the soft linear control system $\left(A, f_{\Sigma(H)}\right.$ on Heisenberg group of dimension 3 with observation as one step down canonical projection to $\mathrm{IR}^{2}$. Then, we have three cases:

Case1

$\pi_{1,2}\left(\begin{array}{ccc}1 & a & c \\ 0 & 1 & b \\ 0 & 0 & 1\end{array}\right)=(a, b)$

Case 2

$\pi_{1,3}\left(\begin{array}{ccc}1 & a & c \\ 0 & 1 & b \\ 0 & 0 & 1\end{array}\right)=(a, c)$

Case 3

$$
\pi_{2,3}\left(\begin{array}{ccc}
1 & a & c \\
0 & 1 & b \\
0 & 0 & 1
\end{array}\right)=(b, c)
$$

According to the characterization given in ref. [2], there is no observable system in any case. Because, $2=\operatorname{dim} S<\operatorname{dim} G=3$ and $S$ is an Ablian Lie group. So, we cannot construct any soft linear control system that is observable.

What we can do is to take $S$ as a nilpotent Lie group of dimension 3 and $h$ as any morphism so that there exists at least one observable system.
So, $f_{\Sigma(H)}$ can assign to them in order to have observable soft linear control system for Heisenberg group.

In ref. [2], it is shown that no observable such system exists on semisimple groups and given necessary conditions for the existence of such a system on a general Lie group.

\section{Conclusion}

In this work, soft theory has been applied to control theory. For this aim, first, we have introduced the notion of soft Lie group and the connection with its soft Lie algebra. We have constructed the usual soft control system on a Lie group and defined the soft linear control system on a Lie group. Linear control systems are important from their application points of view and this work serves a new approach. One can find alternative applications for further work. For intance; how one can construct such a system so that $f_{\Sigma}$ assigns $x$ to the closest observable one. Constructing soft control systems can be useful for their applications on models. In this work, we have presented on the soft linear control system with observability characterization as a decision argument.

\section{References}

1. Ayala V, Hacibekiroglu A (1997) Observable linear pairs. Comput Appl Math 16(3): 205-214.

2. Jouan $F$ (2009) On the Existence of the Observable Linear Systems on Lie Groups. Journal of Dynamical and Control Systems 15(2): 263-276.

3. Ibrahim AM, Yusuf AO (2012) Development of Soft Set Theory. American International Journal of Contemporary Research 2(9).

4. Acar U, Koyuncu F, Tanay B (2010) Soft sets and soft rings. Computers and Mathematics with Applications 59: 3458-3463.

5. Cagman N, Karatas S, Enginoglu S (2011) Soft topology. Computers and Mathematics with Applications 62: 351-358.

6. Molodtsov D (1999) Soft set theory-first results. Computers Mathematics with Applications 37(4-5): 1931.

7. Maji PK Biswas R, Roy AR (2003) Soft set theory. Computers Math Applic 45: 555-562.

8. Maji PK, Roy AR, Biswas R (2002) An application of soft sets in desicion making problem. Comput Math Appl 44: 1077-1083.

9. Shabir M, Naz M (2011) On soft topological spaces. Comput Math Appl 61 1786-1799.

10. Zorlutuna I, Akdag M, Min WK, Atmaca S (2012) Remarks on soft topological spaces. Annals of Fuzzy Mathematics and Informatics 3(2): 171-185.

11. Aktas H, Cagman N (2007) Soft sets and soft groups. Information Sciences 177: $2726-2735$

12. Aktas H, Cagman N (2009) Erratum to Soft sets and soft groups. Information Sciences 179: 338

13. Nazmul S, Samanta SK (2012) Soft topological soft groups. Mathematical Sciences 6: 1-10.

14. Akram M, Feng F (2013) Soft intersection Lie algebras. Quasigroups and Related Systems 21: 1118 